\title{
CHUYỂN MẠCH QUANG HỌC DỤ๋A TRÊN ĐIỀU KHIỂN PHA TRONG HỆ NGUYÊN TỬ BA MÚC LAMBDA CẤU HÌNH VÒNG
}

Nguyễn Tuấn Anh ${ }^{(1)}$, Thái Doãn Thanh ${ }^{(1)}$, Nguyễn Xuân Hào ${ }^{(2)}$, Thái Đình Thịnh ${ }^{(3)}$, Hoàng Minh Đồng ${ }^{(1)}$

(1) Truờng Đại học Công nghiệp Thưc phẩm TP Hồ Chí Minh; (2) Truờng Đại học Thủ Dầu Một;

(3) Truòng Trung học phổ thông Hoàng Mai (Nghệ An)

Liên hệemail:dong.gtvtmt@gmail.com

https://doi.org/10.37550/tdmu.VJS/2020.01.017

\section{Tóm tắt}

Chuyển mạch toàn quang là thành phần quan trọng trong các mạng truyền thông quang học tốc độ cao và có các ứng dụng tiềm năng trong các hệ thống thông tin lương tư và máy tính lương tử. Bài báo này trình bày kết quả nghiên cúu đặc tính hấp thu, tán sắc và sụ chuyển mạch quang học của truờng laser dò thông qua điều khiển pha tương đối của các trương laser điều khiển trong hệ nguyên tủ ba múc cấu hình vòng bằng phuơng pháp giải đồng thời hẹ phuơng trình Maxwell-Bloch đối với nguyên tủ và các trường laser. Chúng tôi kết luận rằng các đặc tính hấp thụ và tán sắc của trường laser dò nhu một hàm của pha tuoong đối $\phi$. Khi điều chinh pha tuơng đối của các trương laser, hàm bao đầu vào của trưòng laser dò là một sóng liên tục sẽ được bật; khi pha tương đối của trương laser được biến điệu có chu kỳ, sóng liên tục sẽ được tắt.

Tù khóa: cấu hình vòng, hệ nguyên tủ, truò̀ng laser

\section{Abstract}

\section{OPTICAL SWITCHING BASED ON THE PHASE-CONTROL IN A CLOSED-LOOP THREE-LEVEL LAMBDA SYSTEM}

All-optical switch is an important component in high-speed optical communication networks and has potential applications in quantum information systems and quantum computing. This article presents the results that absorption - dispersive properties and optical switching behaviors of a probe field via the relative phase control of driving fields in the closed-loop three-level A-type atomic system by method simultaneously numerical solve the coupled Maxwell-Bloch equations for atom and field on a spatiotemporal grid. We conclude that the absorption-gain and dispersive properties of the probe field as a function of relative phase $\phi$. When the modulating the relative phase of the applied fields, the continuous-wave input probe field is switched on; when the relative phase of the applied field is periodically modulated, the continuous-wave input probe field is switched off. 


\section{Giới thiệu}

Chuyển mạch toàn quang là thành phần quan trọng trong các mạng truyền thông quang học tốc độ cao, có ứng dụng tiềm năng trong các hệ thống thông tin lượng tử và máy tính lượng tử (Hiroshi Ishikawa, 2008). Sự ra đời của hiệu ứng trong suốt cảm ứng điện từ (EIT) (Imamoğlu and Harris, 1989; Boller, Imamoglu, Harris, 1991) đã cung cấp cách tiếp cận tối ưu cho việc chuyển đổi quang học ở ánh sáng yếu (Harris and Yamamoto, 1998; Jafarzadeh, 2017). Môi trường EIT không chỉ triệt tiêu hấp thụ tuyến tính (Fleischhauer, Imamoglu, Marangos, 2005; Khoa, Trung, Thuan, Doai and Bang, 2017) mà còn tăng cường độ cảm phi tuyến ở lân cận tần số cộng hưởng nguyên tử (Khoa, Doai, Son and Bang, 2014; Hamedi, Gharamaleki, and Sahrai, 2016), do đó, môi trường EIT có thể tăng cường tương tác giữa ánh sáng và vật chất một cách đáng kể, cho phép các quá trình quang phi tuyến đạt được hiệu quả đáng kể ngay cả ở mức đơn photon (Shiau, $\mathrm{Wu}$, Lin and Chen, 2011; Venkataraman, Saha and Gaeta, 2013). Hiệu ứng EIT cũng có thể thay đổi động học lan truyền của các xung ánh sáng như sự hình thành và lan truyền của các soliton quang (Huang, Jiang, Payne and Deng, 2006; Dong, Doai and Bang, 2018)...

Với cách tiếp cận khác để nghiên cứu điều khiển sự kết hợp và giao thoa lượng tử, pha tương đối của các trường laser ứng dụng đã được sử dụng rộng rãi trong một số quá trình quan trọng trong các hệ thống nguyên tử, phân tử và trạng thái rắn (Korsunsky, Leinfellner, Huss, Baluschev, Windholz, 1999; Zhi Qiang Zeng, Yu-Ping Wang, and Bang Pin Hou, 2013). Đặc biệt trong cấu hình nguyên tử với sơ đồ cấu hình vòng lượng tử, pha tương đối đóng vai trò rất quan trọng trong sự tương tác giữa các trường laser và môi trường. Pha tương đối có thể thay đổi đáng kể các tính chất quang tuyến tính và phi tuyến và dẫn đến một số hiện tượng thú vị như điều khiển pha của EIT (Korsunsky, Leinfellner, Huss, Baluschev, Windholz, 1999; Bortman-Arbiv, Wilson-Gordon, Friedmann, 2001), khuếch đại mà không đảo lộn độ cư trú (Xu, Wu, Gao, 2003), địa phương hóa nguyên tử theo pha tương đối (Chengpu Liu, Shangqing Gong, Takashi Nakajima, Zhizhan Xu, 2006), tăng cường tính phi tuyến Kerr liên quan với sự tự biến điệu pha và biến điệu pha chéo (Fountoulakis, Terzis, Paspalakis, 2010; Sun, Niu, Jin and Gong, 2007, 2008; Qi, Niu, Zhou, Peng and Gong, 2011), hành vi truyền qua (Xu, Wu, Gao, Zhang, 2003; Zhi Qiang Zeng, Yu-Ping Wang, and Bang Pin Hou, 2013), chuyển mạch quang và các đặc tính kết hợp phụ thuộc pha khác (Dong-chao Cheng, Cheng-pu Liu, Shang-qing Gong, 2006; Agarwal, Tarak Nath Dey, Sunish Menon, 2001; Jiahua Li, 2007; Kou, Wan, Kang, Jiang, Wang, Jiang, Gao, 2011; Hoang Minh Dong, and Nguyen Huy Bang, 2019; Giovanna Morigi, Sonja Franke-Arnold, Gian-Luca Oppo, 2002; Hoonsoo Kang, Gessler Hernandez, Jiepeng Zhang, and Yifu Zhu, 2006)... đã được thực hiện cả về lý thuyết và thực nghiệm trong một hệ thống nguyên tử vòng kín.

Trong bài báo này, chúng tôi nghiên cứu ảnh hưởng của pha tương đối của các trường laser liên kết lên hành vi hấp thụ, tán sắc và đặc tính chuyển mạch quang của trường laser dò trong cấu hình vòng kín bởi trường microwave thêm vào kết nối hai trạng 
thái thấp hơn. Trường microwave đóng một vai trò quan trọng trong sơ đồ. Đầu tiên, nó có thể tạo ra sự kết hợp lượng tử giữa mức thấp hơn, việc này có thể điều khiển các tính chất tán sắc và hấp thụ của hệ bằng cách thay đổi cường độ của trường microwave (Dongchao Cheng, Cheng-pu Liu, Shang-qing Gong, 2006; Agarwal, Tarak Nath Dey, Sunish Menon, 2001). Thứ hai, trường microwave biến hệ $\Lambda$ thành vòng kín. Chúng ta biết rằng các hệ vòng kín có đặc tính phụ thuộc pha, tức là, hành vi của hệ chủ yếu phụ thuộc vào pha tương đối giữa các kênh chuyển tiếp (Giovanna Morigi, Sonja Franke-Arnold, GianLuca Oppo, 2002). Sử dụng các hiệu ứng như vậy, chúng ta có thể tạo ra điều khiển pha của hành vi truyền qua và thực hiện chuyển mạch quang học. Ngoài ra, nhiều sự chú ý trong sự kết hợp do trường microwave đã được nghiên cứu (Giovanna Morigi, Sonja Franke-Arnold, Gian-Luca Oppo, 2002). Hầu hết các công trình này liên quan đến hệ nguyên tử điều khiển bằng trường microwave đều tập trung vào đáp ứng ở trạng thái dừng của môi trường. Chúng tôi thấy rằng trong một hệ nguyên tử điều khiển bằng trường microwave như vậy, các tính chất hấp thụ, tán sắc của trường laser dò có thể được điều khiển bởi pha tương đối của các trường laser liên kết. Chúng tôi cũng nhận thấy khi hàm bao ban đầu của trường laser dò là sóng liên tục thì có thể được bật và tắt theo pha tương đối của các trường laser liên kết có chu kỳ theo thời gian, tức là, trạng thái chuyển mạch có thể được điều khiển theo pha tương đối của các trường laser liên kết.

\section{Mô hình và các phương trình cơ bản}

Chúng tôi xem xét hệ nguyên tử ba mức vòng kín cấu hình lambda được liên kết bởi hai trường laser và trường laser điều khiển microwave, như trong hình 1 .

Hình. 1. So đồ năng lượng của hẹ nguyên tư ba múcc vòng kin cấu hình labda.

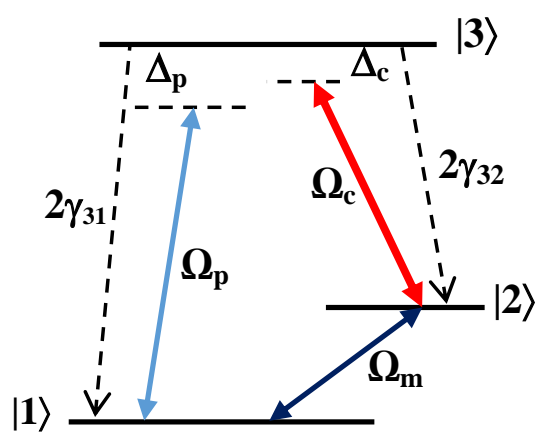

Dịch chuyển $|3\rangle \leftrightarrow|2\rangle$ được điều khiển bởi trường laser điều khiển với tần số Rabi $2 \Omega_{\mathrm{c}}$, pha ban đầu $\varphi_{\mathrm{c}}$ và tần số góc $\omega_{\mathrm{c}}$. Trường laser dò có tần số Rabi $2 \Omega_{\mathrm{p}}$, pha ban đầu $\varphi_{\mathrm{p}}$ và tần số góc $\omega_{\mathrm{p}}$ được đặt vào dịch chuyển $|3\rangle \leftrightarrow|1\rangle$. Trường điều khiển microwave với tần số Lamor $2 \Omega_{\mathrm{m}}$, pha ban đầu $\varphi_{\mathrm{m}}$ và tần số góc $\omega_{\mathrm{m}}$ được sử dụng để liên kết các mức dưới Zeeman $|2\rangle$ và $|1\rangle$ thông qua dịch chuyển lưỡng cực từ được phép và để tạo thành cấu hình vòng kín. Vai trò ban đầu của trường microwave là tạo ra sự kết hợp lượng tử giữa các mức $|2\rangle$ và $|1\rangle$, cần thiết cho các hiệu ứng phụ thuộc pha. Một vai trò khác của trường microwave là chuyển nguyên tử từ mức $|1\rangle$ đến mức $|2\rangle$ để có thể tiếp tục được 
bơm vào mức $|3\rangle$ bằng trường laser điều khiển $\omega_{\mathrm{c}}$. Tốc độ phân rã tự phát từ mức $|3\rangle$ xuống mức $|1\rangle$ và $|2\rangle$ tương ứng là $\gamma_{31}$ và $\gamma_{32}$. Trong bức tranh tương tác, với gần đúng sóng xoay và gần đúng lưỡng điện cực, Hamiltonian bán cổ điển mô tả tương tác giữa nguyên tử và trường laser cho hệ đang xét có thể được viết là (với giả định $\hbar=1$ ):

$$
H_{\text {int }}=\left[\begin{array}{ccc}
-\Delta_{p} & -\Omega_{m}^{*} \mathrm{e}^{i \phi} & -\Omega_{p}^{*} \\
-\Omega_{m} \mathrm{e}^{-i \phi} & -\Delta_{c} & -\Omega_{c}^{*} \\
-\Omega_{p} & -\Omega_{c} & 0
\end{array}\right],
$$

ở đây $\Delta_{p}=\omega_{31}-\omega_{p}, \Delta_{c}=\omega_{32}-\omega_{c}$, và $\phi=\left(\omega_{c}+\omega_{m}-\omega_{p}\right) t+\left(\varphi_{c}+\varphi_{m}-\varphi_{p}\right)$ là các độ lệch tần của trường laser dò và trường laser điều khiển, tương ứng. Sự tiến triển động học của hệ có thể được mô tả bởi phương trình Liouville:

$$
\frac{\partial \rho}{\partial t}=-i\left[H_{\mathrm{int}}, \rho\right]+\Lambda \rho,
$$

và các phương trình ma trận mật độ có liên quan thu được cho hệ lambda ba mức vòng kín được đưa ra như sau:

$$
\begin{aligned}
& \frac{\partial \rho_{11}}{\partial t}=2 \gamma_{31} \rho_{33}+i \Omega_{p}^{*} \rho_{31}-i \Omega_{p} \rho_{13}+i \Omega_{m}^{*} e^{i \phi} \rho_{21}-i \Omega_{m} e^{-i \phi} \rho_{12}, \\
& \frac{\partial \rho_{22}}{\partial t}=2 \gamma_{32} \rho_{33}-i \Omega_{m}^{*} e^{i \phi} \rho_{21}+i \Omega_{m} e^{-i \phi} \rho_{12}+i \Omega_{c}^{*} \rho_{32}-i \Omega_{c} \rho_{23}, \\
& \frac{\partial \rho_{33}}{\partial t}=-2\left(\gamma_{31}+\gamma_{32}\right) \rho_{33}-i \Omega_{p}^{*} \rho_{31}+i \Omega_{p} \rho_{13}-i \Omega_{c}^{*} \rho_{32}+i \Omega_{c} \rho_{23}, \\
& \frac{\partial \rho_{21}}{\partial t}=\left(i\left(\Delta_{c}-\Delta_{p}\right)-\gamma_{21}\right) \rho_{21}-i \Omega_{m} e^{-i \phi}\left(\rho_{22}-\rho_{11}\right)-i \Omega_{p} \rho_{23}+i \Omega_{c}^{*} \rho_{31}, \\
& \frac{\partial \rho_{31}}{\partial t}=-\left(i \Delta_{p}+\gamma_{31}+\gamma_{32}\right) \rho_{31}-i \Omega_{p}\left(\rho_{33}-\rho_{11}\right)+i \Omega_{c} \rho_{21}-i \Omega_{m} e^{-i \phi} \rho_{32}, \\
& \frac{\partial \rho_{32}}{\partial t}=-\left(i \Delta_{c}+\gamma_{31}+\gamma_{32}\right) \rho_{32}+i \Omega_{p} \rho_{12}-i \Omega_{c}\left(\rho_{33}-\rho_{22}\right)-i \Omega_{m}^{*} e^{i \phi} \rho_{31},
\end{aligned}
$$

trong đó, các phần tử ma trận tuân theo các điều kiện liên hợp và chuẩn hóa, cụ thể là $\rho_{i j}=\rho_{i j}^{*}(i \neq j)$, và $\rho_{11}+\rho_{22}+\rho_{33}=1$, tương ứng.

Để nghiên cứu động học của các xung laser lan truyền trong môi trường, phương trình sóng Maxwell dưới gần đúng hàm bao biến thiên chậm và gần đúng sóng quay, chúng ta thu được phương trình sóng đối với trường laser dò:

$$
\frac{\partial \Omega_{p}(z, t)}{\partial z}+\frac{1}{c} \frac{\partial \Omega_{p}(z, t)}{\partial t}=i \alpha \gamma_{21} \rho_{21}(z, t),
$$

ở đây, $\alpha=\frac{\omega_{p} N\left|d_{21}\right|^{2}}{4 \varepsilon_{0} c \hbar \gamma_{21}}$, là hằng số lan truyền. Để thuận tiện, chúng ta thực hiện trong hệ quy chiếu với $\xi=\mathrm{z}$ và $\tau=t-z / c$, theo đó ta có $\partial / \partial z=\partial / \partial \xi-1 / c \partial / \partial \tau, \partial / \partial t=\partial / \partial \tau$, các phương trình ma trận mật độ Bloch $(3 \mathrm{a})-(3 \mathrm{f})$ đối với các phần tử, $\rho_{i j}(\xi, \tau)$ và phương trình sóng Maxwell (4) có thể viết lại như sau: 


$$
\begin{aligned}
& \frac{\partial \rho_{11}}{\partial \tau}=2 \gamma_{31} \rho_{33}+i \Omega_{p}^{*} \rho_{31}-i \Omega_{p} \rho_{13}+i \Omega_{m}^{*} e^{i \phi} \rho_{21}-i \Omega_{m} e^{-i \phi} \rho_{12}, \\
& \frac{\partial \rho_{22}}{\partial \tau}=2 \gamma_{32} \rho_{33}-i \Omega_{m}^{*} e^{i \phi} \rho_{21}+i \Omega_{m} e^{-i \phi} \rho_{12}+i \Omega_{c}^{*} \rho_{32}-i \Omega_{c} \rho_{23}, \\
& \frac{\partial \rho_{33}}{\partial \tau}=-2\left(\gamma_{31}+\gamma_{32}\right) \rho_{33}-i \Omega_{p}^{*} \rho_{31}+i \Omega_{p} \rho_{13}-i \Omega_{c}^{*} \rho_{32}+i \Omega_{c} \rho_{23}, \\
& \frac{\partial \rho_{21}}{\partial \tau}=\left(i\left(\Delta_{c}-\Delta_{p}\right)-\gamma_{21}\right) \rho_{21}-i \Omega_{m} e^{-i \phi}\left(\rho_{22}-\rho_{11}\right)-i \Omega_{p} \rho_{23}+i \Omega_{c}^{*} \rho_{31}, \\
& \frac{\partial \rho_{31}}{\partial \tau}=-\left(i \Delta_{p}+\gamma_{31}+\gamma_{32}\right) \rho_{31}-i \Omega_{p}\left(\rho_{33}-\rho_{11}\right)+i \Omega_{c} \rho_{21}-i \Omega_{m} e^{-i \phi} \rho_{32}, \\
& \frac{\partial \rho_{32}}{\partial \tau}=-\left(i \Delta_{c}+\gamma_{31}+\gamma_{32}\right) \rho_{32}+i \Omega_{p} \rho_{12}-i \Omega_{c}\left(\rho_{33}-\rho_{22}\right)-i \Omega_{m}^{*} e^{i \phi} \rho_{31}, \\
& \frac{\partial \Omega p}{\partial(\alpha \xi)}=i \gamma_{31} \rho_{31}(\xi, \tau) .
\end{aligned}
$$

\section{Kết quả và thảo luận}

Trước hết, chúng tôi xét ảnh hưởng của pha tương đối $\phi$ đến các tính chất hấp thụ và tán sắc của trường laser dò. Để đơn giản, chúng tôi giả sử rằng mỗi trường đặt vào giữa hai mức là cộng hưởng với các dịch chuyển nguyên tử tương ứng, cụ thể là, $\omega_{c}+\omega_{m}-\omega_{p}=0$ và $\Delta_{\mathrm{p}}=\Delta_{\mathrm{c}}=0$. Vì vậy pha tương đối chỉ liên quan tới pha ban đầu của các trường được đặt vào $\phi=\varphi_{c}+\varphi_{m}-\varphi_{p}$.

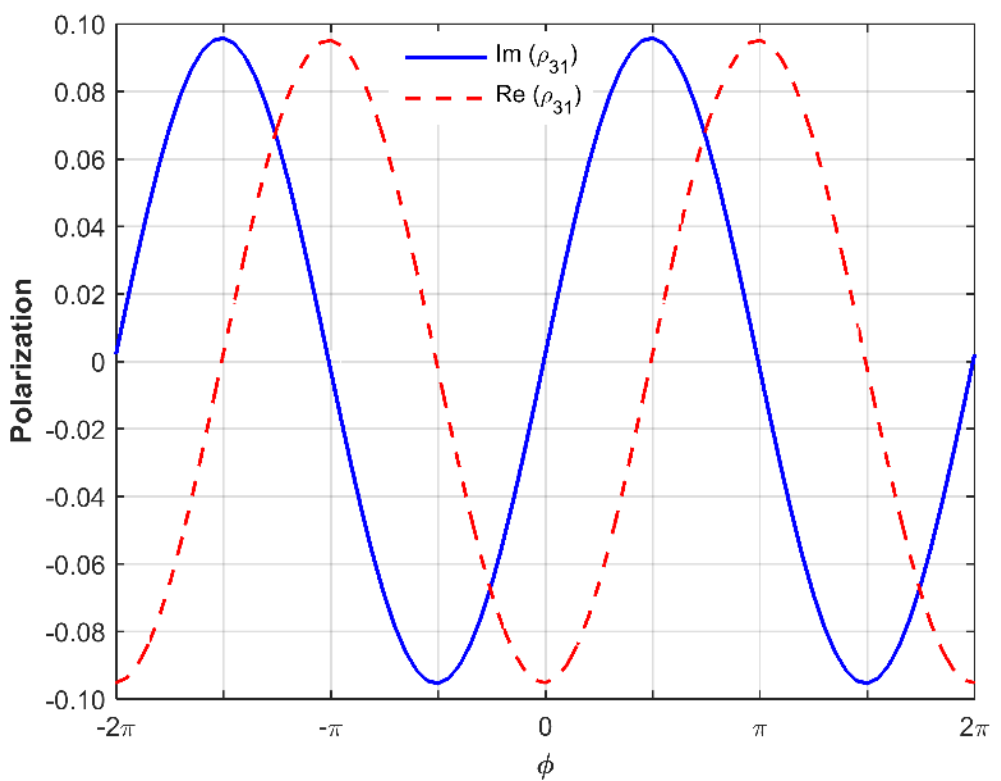

Hình. 2. Các hệ số hấp thụ $\operatorname{Im}\left(\rho_{31}\right)$ (đường màu xanh nét liền) và tán sắc $\operatorname{Re}\left(\rho_{31}\right)$ (đưòng màu đỏ đưt nét) theo pha tương đối $\phi$. Các tham số được chọn $\Omega_{p}=0.5 \gamma_{31}, \Omega_{c}=$ $10 \gamma_{31}, \Omega_{m}=1 \gamma_{31}, \Delta_{p}=0, \Delta_{c}=0$, và $\gamma_{32}=\gamma_{31}$, tuoong úng. 
Trong hình 2 , chúng tôi vẽ hấp thụ $\operatorname{Im}\left(\rho_{31}\right)$ và tán sắc $\operatorname{Re}\left(\rho_{31}\right)$ của trường dò theo pha tương đối $\phi$. Từ hình 2 , chúng ta có thể thấy rằng $\operatorname{Im}\left(\rho_{31}\right)$ và $\operatorname{Re}\left(\rho_{31}\right)$ biểu hiện các chu kỳ âm, dương với chu kỳ $2 \pi$. Khi pha tương đối $\phi=k \pi$ ( $\mathrm{k}$ là số nguyên), chẳng hạn $\phi=0$ và $\phi=\pi$, hấp thụ bằng không với chiết suất lớn nhất (tán sắc), trong suốt hoàn toàn có thể đạt được. Khi pha tương đối $\phi=(2 k+1) \pi / 2$, hệ chuyển từ hấp thụ tới khuếch đại và ngược lại, cụ thể; đối với $\phi=2 k \pi+\pi / 2$ chẳng hạn $\phi=\pi / 2$ giá trị đỉnh của hấp thụ xảy ra tại vạch tán sắc bằng không; và $\phi=2 k \pi+3 \pi / 2$ chẳng hạn $\phi=3 \pi / 2$ giá trị đỉnh của khuếch đại dò có thể xuất hiện tại vạch tán sắc không. Do đó hệ ba mức cấu hình vòng kín dạng lambda có thể được sử dụng cho chuyển mạch pha của hấp thụ và (hoặc) khuếch đại trường dò.

Trong phần sau, chúng tôi giải số các phương trình $(5 \mathrm{a})$ - $(5 \mathrm{~g})$ trên lưới thời gian bằng phương pháp Runge-Kutta bậc bốn và phương pháp sai phân hữu hạn đối với điều kiện ban đầu là tất cả các nguyên tử ở trạng thái cơ bản $|1\rangle$ và điều kiện biên ban đầu của trường laser dò được giả sử là một sóng liên tục $(\mathrm{cw})$ tại đầu vào môi trường.

(a)

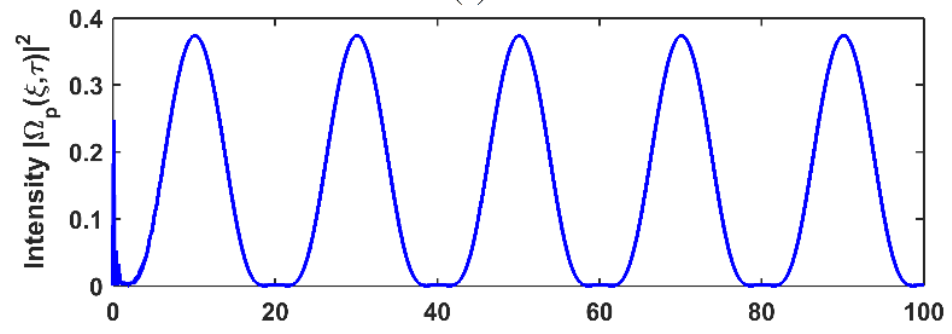

(b)

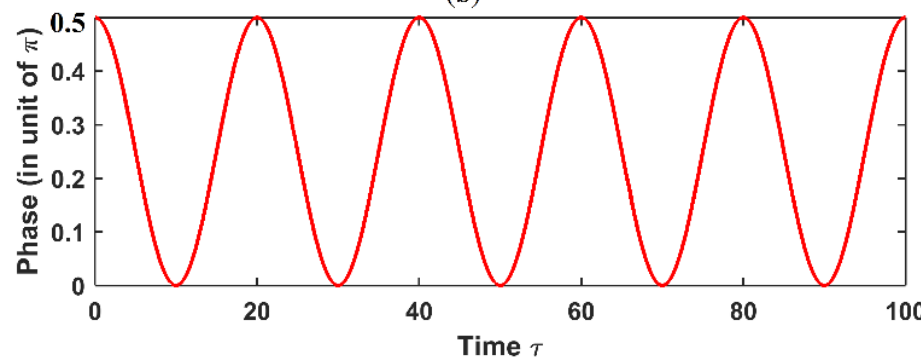

Hình. 3. (a) Sự tiến triển truờng dò liên tuc tại vị trí $\xi=50 / \alpha$ theo sư biến thiên của pha tuoong đối $\phi(\tau)$; $(b)$ pha tuoong đối $\phi(\tau)$ theo thời gian là sóng dạng cosin trong miền tù̀ 0 tới $\pi / 2: \phi(\tau)=\pi / 4 \cos (2 \pi f \tau)+\pi / 4$. Các tham số khác được chọn $\Omega_{p}=0.5 \gamma_{31}, \Omega_{c}=$ $10 \gamma_{31}, \Omega_{m}=1 \gamma_{31}, f=0.05 \gamma_{31}, \Delta_{p}=0, \Delta_{c}=0$, và $\gamma_{32}=\gamma_{31}$, tuoong úng.

Hình 3 cho thấy quá trình chuyển mạch có thể của trường laser lan truyền thông qua môi trường ba mức cấu hình lambda vòng kín tại vị trí $\xi=50 / \alpha$. Hình $3 \mathrm{a}$, cho thấy rằng sự truyền của cường độ trường laser dò như là hàm của thời gian dưới sự biến thiên của pha tương đối $\phi(\tau)$. Sự biến điệu của sóng loại cosin trong miền từ 0 tới $\pi / 2: \not(\tau)=\pi / 4 \cos (2 \pi f \tau)$ $+\pi / 4$ như thấy trên hình $3 b$, mà được tạo ra bởi điện áp cosin đưa vào bộ biến điệu quang- 
điện (EOM) (Hoonsoo Kang, Gessler Hernandez, Jiepeng Zhang, and Yifu Zhu, 2006). Hình $4 \mathrm{a}$ vẽ sự truyền qua của cường độ trường dò như là hàm của thời gian và pha tương đối $\phi(\tau)$ theo thời gian là một sóng gần vuông với sườn trước, sườn sau là trơn tròn trong miền từ 0 tới $\pi / 2: \phi(\tau)=\pi / 2\{1-0.5 \tanh (0.4(\tau-10))+0.5 \tanh (0.4(\tau-35))-$ $0.5 \tanh (0.4(\tau-60))+0.5 \tanh (0.4(\tau-85))\}$, mà được chuyển hóa bởi $\pi$ trong hình $4 \mathrm{~b}$.

(a)

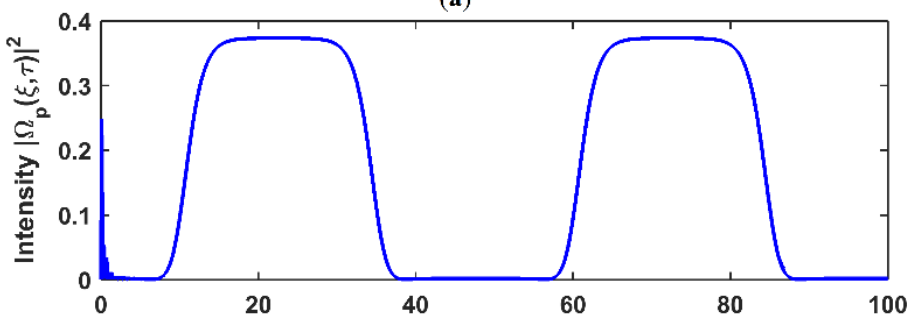

(b)

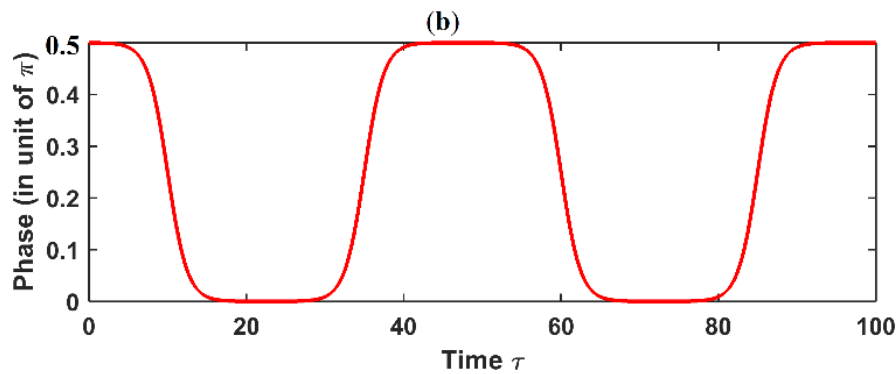

Hinh 4. (a) Su triến triển của trừ̀ng laser dò liên tục tại vị trí $\xi=50 / \alpha$ theo sụ biến thiên của pha tưong đối $\phi(\tau)$; $(b)$ pha tuoong đối $\phi(\tau)$ theo thời gian là một sóng gần

vuông với sườn trước và sau tròn trơn trong miền tù̀ 0 tới $\pi / 2: \phi(\tau)=\pi / 2\{1-$ $0.5 \tanh (0.4(\tau-10))+0.5 \tanh (0.4(\tau-35))-0.5 \tanh (0.4(\tau-60))+0.5 \tanh (0.4(\tau-85))\}$. Các tham số khác chọn như trong hình 3.

Như được biểu hiện trong hình 3 và 4 , sự chuyển mạch có chu kỳ của ánh sáng laser dò lan truyền so với pha tương đối $\phi$ có thể được hình thành một cách hiệu quả theo cách không đồng bộ đối với sự thay đổi có chu kỳ của pha tương đối và quá trình chuyển mạch được đảo ngược thông qua tại pha tương đối bằng $\pi / 2$. Sự truyền của trường laser dò là có thể hoặc "bật" hoặc "tắt" với sự chuyển đổi của pha tương đối. Những kết quả này có thể được giải thích tốt trong các số hạng được mô tả ở trên của tín hiệu dò như thấy trong hình 2 , trong đó sự hấp thụ hoặc khuếch đại và tán sắc thu được tùy thuộc vào giá trị pha tương đối.

\section{Kết luận}

Chúng tôi đã nghiên cứu sự hấp thụ (khuếch đại), tán sắc và hành vi chuyển mạch quang của xung laser dò trong hệ nguyên tử ba mức cấu hình vòng kín được điều khiển bằng trường microwave. Chúng tôi thấy rằng do sự kết hợp lượng tử giữa hai mức thấp hơn của trường microwave gây ra, sự hấp thụ (khuếch đại) và tán sắc của trường dò có thể liên hệ với pha tương đối của các trường laser đặt vào. Bằng cách điều chỉnh pha 
tương đối của các trường laser liên kết, các đặc tính hấp thụ và tán sắc của trường laser dò có thể được thay đổi đáng kể, sự hấp thụ và tán sắc có thể được loại bỏ chỉ bằng cách điều chỉnh pha tương đối vào các vùng thích hợp. Chúng tôi cũng nhận thấy rằng với xung đầu vào của trường laser dò là sóng liên tục được sẽ được "bật" và "tắt" theo pha tương đối của các trường laser khi pha tương đối điều biến theo chu kỳ. Đặc tính hấp thụ đầu xung dò bởi điều khiển pha cung cấp khả năng thực hiện hoạt động chuyển mạch điều khiển pha, mà có thể có ứng dụng trong chuyển mạch quang và các thiết bị khuếch đại quang trong truyền thông quang học.

\section{TÀI LIỆU THAM KHẢO}

[1] Hiroshi Ishikawa (2008), Ultrafast All-Optical Signal Processing Devices, John Wile \& Sons, Singapore.

[2] A. Imamoğlu and S. E. Harris (1989), "Lasers without inversion: interference of dressed lifetime broadened states", Opt. Lett 1344-1346.

[3] K.J. Boller, A. Imamoglu, S.E. Harris (1991), Observation of electromagnetically induced transparency, Phys. Rev. Lett. 66-2593.

[4] Harris S E and Yamamoto Y (1998), Photon Switching by Quantum Interference, Phys. Rev. Lett. 81-3611.

[5] H. Schmidt, R.J. Ram (2000), All-optical wavelength converter and switch based on electromagnetically induced transparency, Appl. Phys. Lett. 76-3173.

[6] A. Fountoulakis, A.F. Terzis, E. Paspalakis (2010), All-optical modulation based on electromagnetically induced transparency, Phys. Lett. A 374-3354.

[7] M. Yan, E.G. Rickey, Y. Zhu (2001), Observation of absorptive photon switching by quantum interference, Phys. Rev. A 64-041801(R).

[8] M.A. Antón, O.G. Calderón, S. Melle, I. Gonzalo, F. Carreño (2006), All-optical switching and storage in a four-level tripod-type atomic system, Opt. Commun. 268-146.

[9] Hoang Minh Dong, Luong Thi Yen Nga, and Nguyen Huy Bang (2019), Optical switching and bistability in a degenerated two-level atomic medium under an external magnetic field, Applied Optics, 58, 4192.

[10] Jiteng Sheng, Xihua Yang, Utsab Khadka and Min Xiao (2011), All-optical switching in an N-type foul-level atom-cavity system, Optics Express 19(18)-17059-64.

[11] H Jafarzadeh (2017), All-optical switching in an open V-type atomic system, Laser Phys. 27-025204.

[12] M. Fleischhauer, A. Imamoglu, J.P. Marangos (2005), Electromagnetically induced transparency: optics in coherent media, Rev. Mod. Phys. 77-633.

[13] Doai L V, Trong P V, Khoa D X, and Bang N H (2014), Electromagnetically induced transparency in five-level cascade scheme of ${ }^{85} \mathrm{Rb}$ atoms: An analytical approach, Optik 125-3666.

[14] Khoa D X, Trung L C, Thuan P V, Doai L V and Bang N H (2017), Measurement of dispersive profile of a multi-window EIT spectrum in a Doppler-broadened atomic medium, $J$. 
Opt. Soc. Am. B. 34-1255.

[15] Khoa D X, Doai L V, Son D H and Bang N H (2014), Enhancement of self-Kerr nonlinearity via electromagnetically induced transparency in a five-level cascade system: an analytical approach, J. Opt. Soc. Am. B. 31-1330.

[16] L.V. Doai, D.X. Khoa, N.H. Bang (2015), EIT enhanced self-Kerr nonlinearity in the threelevel lambda system under Doppler broadening, Phys. Scr. 90-045502.

[17] H. R. Hamedi, A. H. Gharamaleki, and M. Sahrai (2016), Colossal Kerr nonlinearity based on electromagnetically induced transparency in a five-level double-ladder atomic system, Applied Optics 22-5892.

[18] Shiau B-W, Wu M-C, Lin C-C and Chen Y-C (2011), Low-Light-Level Cross-Phase Modulation with Double Slow Light Pulses, Phys. Rev. Lett. 106-193006

[19] Venkataraman V, Saha K and Gaeta A L (2013), Phase modulation at the few-photon level for weak-nonlinearity-based quantum computing, Nat. Photon. 7-138-41.

[20]Huang G, Jiang K, Payne M G and Deng L (2006), Formation and propagation of coupled ultraslow optical soliton pairs in a cold three-state double- $\Lambda$-system, Phys. Rev. E 73-056606.

[21]R. Yu, J. Li, P. Huang, A. Zheng, X. Yang (2009), Dynamic control of light propagation and optical switching through an RF-driven cascade-type atomic medium, Phys. Lett. A 373-2992.

[22] Liu-Gang Si, Xin-You Lu, Xiangying Hao and Jia-Hua Li (2010), Dynamical control of soliton formation and propagation in a Y-type atomic system with dual ladder-type electromagnetically induced transparency, J. Phys. B: At. Mol. Opt. Phys. 43-065403.

[23] Rong Yu, Jiahua Li, Chunling Ding, Xiaoxue Yang (2011), Dual-channel all-optical switching with tunable frequency in a five-level double-ladder atomic system, Opt. Commun. 284-2930.

[24] Yang Chen, Zhengyang Bai, and Guoxiang Huang (2014), Ultraslow optical solitons and their storage and retrieval in an ultracold ladder-type atomic system, Phys. Rev. A 89-023835.

[25] H. M. Dong, L. V. Doai, V. N. Sau, D. X. Khoa and N. H. Bang (2016), Propagation of laser pulse in a three-level cascade atomic medium under conditions of electromagnetically induced transparency, Photonics Letter Poland, 3-73.

[26] D. X. Khoa, H. M. Dong, L. V. Doai and N. H. Bang (2017), Propagation of laser pulse in a three-level cascade inhomogeneously broadened medium under electromagnetically induced transparency conditions, Optik 131-497.

[27] H R Hamedi (2017), Optical switching, bistability and pulse propagation in five-level quantum schemes, Laser Phys. 27-066002.

[28] H M Dong, L V Doai, and N H Bang (2018), Pulse propagation in an atomic medium under spontaneously generated coherence, incoherent pumping, and relative laser phase, Opt. Commun. 426-553-557.

[29] E.A. Korsunsky, N. Leinfellner, A. Huss, S. Baluschev, L. Windholz (1999), Phasedependent electromagnetically induced transparency, Phys. Rev. A 59, 2302.

[30] D. Bortman-Arbiv, A.D. Wilson-Gordon, H. Friedmann (2001), Phase control of group velocity: From subluminal to superluminal light propagation, Phys. Rev. A 63, 043818.

[31]W.H. Xu, J.H. Wu, J.Y. Gao (2003), Phase-dependent properties in a microwave-driven V 
system: gain without population inversion and narrow resonances, Opt. Commun. 223-367-373.

[32] Chengpu Liu, Shangqing Gong, Takashi Nakajima, Zhizhan Xu (2006), Phase-sensitive atom localization in a loop $\Lambda$-system, Journal of Modern Optics, 12-1791-1802.

[33] Sun H, Niu Y P, Jin S Q and Gong S Q (2007), Phase control of cross-phase modulation with electromagnetically induced transparency, J. Phys. B: At. Mol. Opt. Phys. 40-3037.

[34] Sun H, Niu Y P, Jin S Q and Gong S Q (2008), Phase control of the Kerr nonlinearity in electromagnetically induced transparency media, J. Phys. B: At. Mol. Opt. Phys. 41065504.

[35] Y. Qi, Y Niu, F Zhou, Y Peng and S Gong (2011), Phase control of coherent pulse propagation and switching based on electromagnetically induced transparency in a fourlevel atomic system, J. Phys. B: At. Mol. Opt. Phys. 44-085502.

[36] W.H. Xu, J.H. Wu, J.Y. Gao, B. Zhang (2003), Elimination of the transient absorption in a microwave-driven $\Lambda$-type atomic system, Phys. Lett. A 314-23-28.

[37]Zhi Qiang Zeng, Yu-Ping Wang, and Bang Pin Hou (2013), Amplitude and phase control of transient absorption and dispersion in a microwave-driven atomic system, Eur. Phys. J. D 67

[38] Dong-chao Cheng, Cheng-pu Liu, Shang-qing Gong (2006), Optical bistability via amplitude and phase control of a microwave field, Opt. Commun. 263-111-115.

[39] G. S. Agarwal, Tarak Nath Dey, Sunish Menon (2001), Knob for changing light propagation from subluminal to superluminal, Phys. Rev. A, 64-053809.

[40] Jiahua Li (2007), Coherent control of optical bistability in a microwave-driven V-type atomic system, Physica D 228-148-152.

[41] J. Kou, R.G. Wan, Z.H. Kang, L. Jiang, L. Wang, Y. Jiang, J.Y. Gao (2011), Phasedependent coherent population trapping and optical switching, Phys. Rev. A 84-063807.

[42] Hoang Minh Dong, and Nguyen Huy Bang (2019), Controllable optical switching in a closed-loop three-level lambda system, Phy. Scr. 94, 115510.

[43] Giovanna Morigi, Sonja Franke-Arnold, Gian-Luca Oppo (2002), Phase-dependent interaction in a four-level atomic configuration, Phys. Rev. A 66-053409.

[44] Hoonsoo Kang, Gessler Hernandez, Jiepeng Zhang, and Yifu Zhu (2006), Phase-controlled light switching at low light levels, Phys. Rev. A 73-011802(R).

[45] J.H. Wu, J.Y. Gao (2003), Phase and amplitude control of the inversionless gain in a microwave-driven $\Lambda$-type atomic system, Eur. Phys. J. D 23-315-319. 